\title{
Motivationally Significant Self-control: Enhanced Action Withholding Involves the Right Inferior Frontal Junction
}

\author{
David A. O'Connor, Daniel J. Upton, Jennifer Moore, and Robert Hester
}

\begin{abstract}
In everyday life, people use self-control to withhold actions. This ability is particularly important when the consequences of action withholding have an impact on the individual's wellbeing. Despite its importance, it is unclear as to how the neural nodes implicated in action withholding contribute to this realworld type of self-control. By modifying an action withholding paradigm, the go/no-go task, we examined how the brain exerts self-control during a scenario in which the implications of withholding an action are meaningful and motivationally significant. A successfully withheld response contributed to long-term monetary rewards, whereas failure to withhold a response incurred an immediate monetary punishment. Compared with neutral action withholding, participants significantly improved
\end{abstract}

\section{INTRODUCTION}

Although the predominantly right-lateralized frontoparietal neural network associated with the ability to dynamically inhibit inappropriate responses is well established (Criaud \& Boulinguez, 2013; Swick, Ashley, \& Turken, 2011; Rubia et al., 2001), the functional nature of its key nodes remains uncertain. In particular, it is less clear what the relative contributions of nodes in this network are when the need for successful response inhibition is especially motivationally significant. In reality, people often encounter moments in which it is prudent to withhold from acting upon a stimulus, even if it is alluring, because of the divergent and meaningful implications that follow. For example, the impulse to buy another alcoholic drink may seem alluring, despite the knowledge that abstaining from its consumption will promote long-term benefits (e.g., money savings, improved health and well-being), whereas a failure to abstain would incur more immediate and tangible costs (e.g., loss of money, possible social disinhibition). This type of self-control, which is critical for flexible and adaptive interaction with the demands of a changing environment, is of particular clinical relevance to populations characterized by risky impulsive behaviors (e.g., substance use disorder; Kreek, Nielsen, Butelman, \& LaForge, 2005;

University of Melbourne, Victoria, Australia

(C) Massachusetts Institute of Technology their performance when these contingencies were applied. Crucially, although the right IFG and pre-SMA were found to promote overall action withholding, the enhancement in behavioral performance relative to a neutral condition was only reflected by a physiological change in a region encompassing the right inferior frontal junction and precentral gyrus. We speculate that the ability to flexibly modulate attention to goalrelevant stimuli is crucial to enhanced, motivationally driven action withholding and that this ability is subserved by the right inferior frontal junction. These findings suggest that controlmodulating factors, rather than action withholding processes per se, can be critical to improving motivationally significant action withholding outcomes.

Robbins \& Everitt, 1999), who demonstrate a compromised capacity to stop inappropriate actions (Fillmore \& Rush, 2002; Salo et al., 2002) despite their negative consequences (Goldstein et al., 2009).

Given its relevance, we aimed to model this type of inhibitory control behavior using a modified go/no-go task. A delayed-reward/immediate-punishment (DR-IP) outcome scenario was employed, which required participants to implement inhibitory control over a motor response for trials with previously learned response-reward associations. Similar to the long-term benefits brought about by successful abstinence over immediately rewarding stimuli in the real world, successful inhibition on these trials contributed to a larger monetary reward-the feedback for which was DR. However, unsuccessful inhibition for trials with previously learned response-reward associations led to a monetary punishment with immediate and tangible costs (IP).

We hypothesized that the DR-IP condition would be associated with improved inhibitory control in a group of healthy control participants compared with a task condition without such DR-IP contingencies (neutral). We further hypothesized that a predominantly right-lateralized frontoparietal neural network including the right IFG (rIFG) and pre-SMA would support successful inhibition (Chikazoe et al., 2009; Aron, Fletcher, Bullmore, Sahakian, \& Robbins, 2003; Rubia et al., 2001). Crucially, we also 
hypothesized differences in the relative contributions of this network as a function of task condition (DR-IP vs. neutral). The act of behavioral stopping is commonly attributed to be dependent on rIFG activity (Neubert, Mars, Buch, Olivier, \& Rushworth, 2010; Forstmann et al., 2008; Aron, 2006; Garavan, Ross, \& Stein, 1999), which has been characterized as a brake that can suppress motor responses in a variety of contexts (Aron, Robbins, \& Poldrack, 2014). Thus, if anticipated improvement in DR-IP performance is attributable to the rIFG, then activity should be modulated in this region. Recent work, however, suggests that variation in inhibitory control performance may not always be exclusively attributable to behavioral stopping processes per se. Variation in performance may instead be mediated by other mechanisms related to processes such as salience, attention, and motivation (Salinas \& Stanford, 2013; Wiecki \& Frank, 2013; Chatham et al., 2012; Boehler, Appelbaum, Krebs, Chen, \& Woldorff, 2011). As a consequence, modulation of DR-IP performance may be more closely associated with other neural nodes in the inhibitory control network, which are implicated in stimulus-driven and goal-directed attention such as the right inferior frontal junction (rIFJ; Corbetta \& Shulman, 2011; Asplund, Todd, Snyder, \& Marois, 2010; Brass, Derrfuss, Forstmann, \& Cramon, 2005).

We also incorporated trials that did not require action withholding but rather provided immediate monetary rewards for rapid responding (money trials). We examined activity in nodes associated with action withholding during these money trials for two reasons: first, to uncouple activity related to action withholding from general task-related activity pertaining to attention; second, to ascertain whether nodes associated with improved DR-IP performance may also show sensitivity to context. That is, in addition to action withholding, are some nodes also modulated by other types of trials that may exert an influence stopping performance, such as money trials? In addition, given our interest in attention, we employed faces as stimuli so that sensitivity could be observed in an extrastriate visual region susceptible to attentional modulation (fusiform face area [FFA]; Egner \& Hirsch, 2005; O'Craven \& Kanwisher, 2000).

\section{METHODS}

\section{Participants}

Seventeen healthy volunteers (five men, mean age = 25 years) took part in the current study. All of the participants were right handed, as determined by the Edinburgh Handedness Inventory (Oldfield, 1971), and reported no current or past history of neurological or psychiatric disorders or psychotropic medication use. Participants provided informed consent, and procedures were carried out in compliance with ethical research institutional guidelines.

\section{Behavioral Task}

In the current modified go/no-go task (Figure 1), participants were presented with images of famous faces. Single button presses were required upon presentation of all faces on the screen except one, the no-go trial (e.g., Bill Clinton), which was assigned at the beginning of each run. Face stimuli consisted of well-known actors and politicians/world leaders. Prescreening with all participants ensured 100\% recognition before task administration. Participants were instructed that, except for no-go trials, faces of politicians/world leaders were money trials that would provide immediate feedback of a small monetary reward (approximate range $=2 \mathrm{c}-18 \mathrm{c}$ AUD), the magnitude of which was commensurate with their response speed.

As outlined, the task was designed to investigate action withholding over an alluring stimulus that carries divergent implications depending on action withholding success. A DR-IP outcome scenario was modeled by requiring participants to implement inhibitory control of a motor response with previously learned response-reward associations. If successful, the inhibition could contribute to a larger monetary reward with delayed feedback (DR) but, if unsuccessful, would result in immediate monetary punishment (IP).

The reward-response association for DR-IP no-go trials was cultivated by assigning no-go stimuli that had been employed in the previous run as a special go stimulus that provided immediate rewards for rapid responses (i.e., money trials). Money trial stimuli were differentiated by the fact that they were politicians/world leaders. Go trials, on the other hand, were always composed of faces of actors. Participants were informed before the beginning of each run which face was designated as the no-go stimulus and were asked to withhold their button response upon presentation of the no-go stimulus. Rather than receiving an immediate reward for successful inhibitory control of DR-IP no-go trials, participants were provided with monetary reward feedback at the end of each run based on the highest number of consecutive successfully inhibited DR-IP no-go trials during the run, multiplied by 40c. For example, if within one run, the highest number of successful consecutive response inhibitions was seven, then the participant was awarded $\$ 2.80(7 \times 40 \mathrm{c})$. These contingencies were an attempt to model behavior in which abstinence is required over an immediate and tangible reward (i.e., reward amount is based on reaction time with immediate feedback) to obtain a larger yet less tangible reward (i.e., reward amount is based on an accumulation of consecutive inhibitions with no immediate feedback). Failure to inhibit a DR-IP no-go trial, however, resulted in an immediate monetary punishment of 40c (AUD). In addition to three runs of trials consisting DR-IP no-go trials, test sessions included a task condition consisting of no-go trials without a reward-response association or DR-IP contingencies (i.e., neutral). Participants were informed at the beginning of each run whether no-go trials would have DR-IP contingencies. 
Figure 1. DR-IP go/no-go task design. The task required participants to (a) respond rapidly to different famous actors' faces (go trials), (b) respond rapidly to politicians' and world leaders' faces (money trials), and (c) withhold response to a designated face previously employed as a money trial in the preceding run of trials (no-go trials). For money trials, monetary gains were rewarded commensurate with RT speed (maximum gains were 20c), and immediate feedback on performance was provided on screen. For example, an RT of $400 \mathrm{msec}$ for the first depicted money trial (Bill Clinton) results in an immediate reward of $\$ 0.12$ $(1000-400 / 50=12)$. DR-IP contingencies during DR-IP task condition runs (B) were contingent on performance: (a) Successful inhibitory control of DR-IP no-go trials provided a 40c reward; however, rewards were the sum of the longest run of consecutive inhibitions within each run, and feedback for amount held was delayed until the end of the run (DR) For example, if the highest number of consecutive inhibitions was seven, then end-of-run feedback would display $\$ 2.80(\$ 0.40 \times$

$7=\$ 2.80$ ); (b) failure to inhibit

responses resulted in a deduction of 40c from accumulated gains with immediate feedback (IP). DR-IP contingencies did not apply to the neutral task condition (A). Note that the frequency of trial types is for illustrative purposes and is not accurate.
Neutral: No DR-No IP

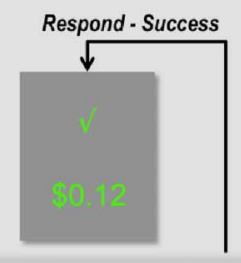

Go Money

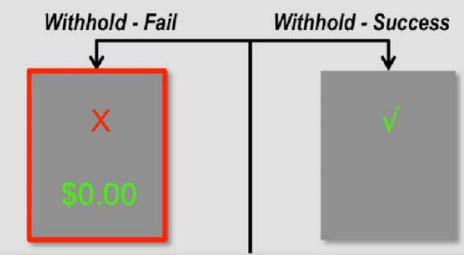

Go

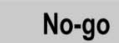

Go

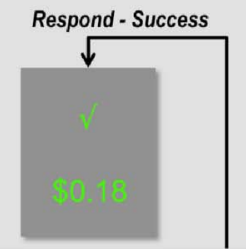

Go

Money
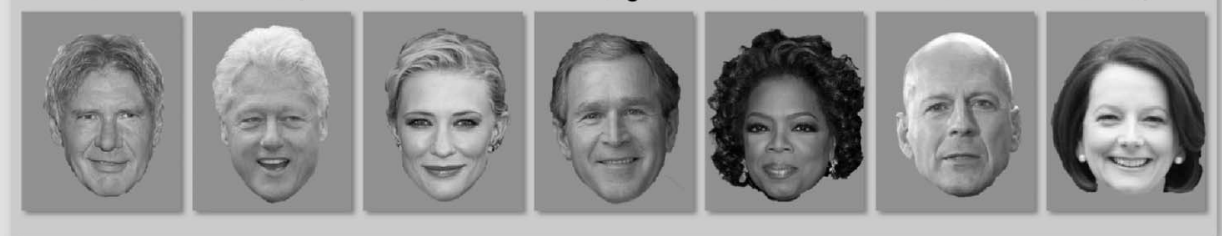

B $D R-I P: D R-I P$
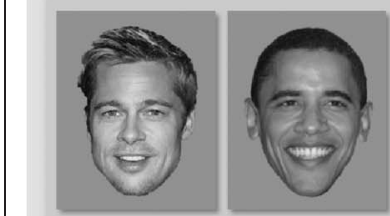

Money
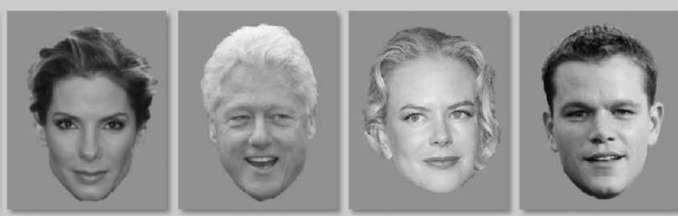

Go
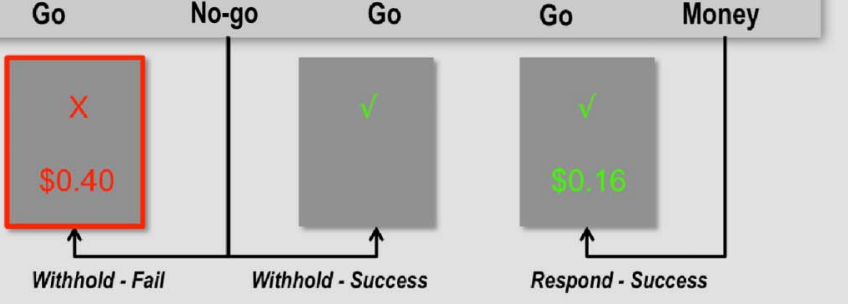

Two different politicians/world leader faces were employed as money trial stimuli for each run. Money trials paid monetary rewards in proportion to how quickly participants responded to their presentation. Successful button presses for a money trial stimulus provided an immediate monetary reward with a maximum value of 20c (AUD). Money trial rewards were calculated by subtracting the reaction time from a 1000-msec response window. For example, if a participant's reaction time was $300 \mathrm{msec}$ in a money trial, the monetary gain would be $14 \mathrm{c}[(1000-300) / 50)=14]$.

The three types of trials, go, money, and no-go trials, presented a face stimulus centrally on a black background for 750 msec. Each run was composed of 120 go, 20 no-go, and 20 money trials presented pseudorandomly such that no-go and money trials were always separated by at least two go trials. Go stimuli were immediately followed by a 1250 -msec ISI presenting only a black background. Money trial stimuli were followed by a feedback screen for $750 \mathrm{msec}$ and blank-screen ISI (500 msec).
No-go trial stimuli were presented for $750 \mathrm{msec}$, followed by a $1250-$ msec ISI, a $1000-m s e c$ feedback screen, and 1000-msec ISI.

All aspects of stimulus delivery and response recording were controlled by E-Prime software (version 2.0; Psychology Software Tools, Pittsburgh, PA), running on a laptop PC (Intel 2 GHz, 256-MB Nvidia Video Card) that was interfaced with the MR scanner during acquisition of fMRI data. Participants indicated their responses by pressing the appropriate button on the MR-compatible response box (Fibre-Optic response pads; Current Designs, Philadelphia, PA). Participants partook in an extensive practice session, which facilitated reward and punishment outcome learning before task performance in the scanner.

\section{Psychometric Testing}

In addition to performance of the behavioral task, all participants completed the Sensitivity to Punishment and Sensitivity to Reward Questionnaire (Torrubia, Ávila, Moltó, 
\& Caseras, 2001). This is a 48-item self-report measure consisting of two scales that assess sensitivity to reward (SR) and sensitivity to punishment (SP). Scales have shown a 3-mont-h test-retest reliability of .87 and .89, respectively (Torrubia et al., 2001), and have also demonstrated good construct validity (Sava \& Sperneac, 2006).

\section{Scanning Parameters}

Functional MR images were acquired at the Royal Children's Hospital, Melbourne, using a 3-T scanner (Siemens Magnetrom TrioTim, Erlangan, Germany). One hundred eighty-three EPI sequences providing T2*-weighted BOLD were acquired for each functional run with the following parameters: repetition time $=2000 \mathrm{msec}$; echo time $=$ 35 msec; flip angle $=90^{\circ} ; 32$ contiguous slices of $4-\mathrm{mm}$ thickness; in-plane resolution $=3.6 \mathrm{~mm} \times 3.6 \mathrm{~mm} \times$ $4 \mathrm{~mm}$. To allow for steady-state tissue magnetization, each functional run began with two volume acquisitions that were later discarded. Six functional runs were collected for each participant. Activation data were registered to high-resolution T1-weighted isotropic $\left(0.8 \mathrm{~mm}^{3}\right)$ structural magnetization-prepared rapid-acquisition gradient echo images to localize the pattern of physiological changes with the task time series.

\section{Data Analysis \\ MRI Data Analysis}

After image reconstruction and concatenation of runs, functional data were time-shifted using Fourier interpolation to remove difference in slice acquisition times, aligned to corresponding anatomical data, and warped to standard Talairach space. Motion was corrected using three-dimensional volume registration with the third volume from the first run as a base. Volumes were blurred using a 4.1-mm FWHM filter. Each voxel was then scaled to a mean of 100, and values over 200 were clipped. All analyses were conducted using AFNI software (afni.nimh. nih.gov/afni/; Cox, 1996).

Separate hemodynamic impulse response functions at 2 -sec temporal resolution were calculated using deconvolution techniques for correct no-go trials and money trials in both DR-IP and neutral task conditions. Response functions for all regressor events were initiated at image acquisition onsets because the presentation of all eventsof-interest was timed to coincide with the beginning of the 2 -sec repetition time cycle. Activity related to trial errors, feedback screens, and motion were also modeled as additional regressors to avoid contamination of baseline and event-related data. Six motion parameters composed of three axes (roll, pitch, and yaw) and three directions (superior, lateral, and posterior) were estimated across the time series for each participant. The baseline estimate was the mean activation recorded during the ongoing trial period (go trials), such that the activa- tion observed during successful no-go and money trial responses represented activation over and above that required for the ongoing trial period. The absence of collinearity between regressors within AFNI X-matrices was verified during the deconvolution procedure using xmat tool.py. After deconvolution, individual beta-coefficient maps for each regressor of interest were extracted, resampled to anatomical data resolution $\left(1 \mathrm{~mm}^{3}\right)$, and masked using a group-averaged EPI mask data set.

Group activation maps for event types (correct DR-IP and correct neutral no-go trials) were determined with one-sample $t$ tests against the null hypothesis of zero event-related activation changes (i.e., no change relative to baseline). Significant voxels passed (a) a voxelwise statistical threshold $(t=5.13, p<.0001)$ and (b) a cluster threshold (part of a larger 162- $\mu$ l cluster of contiguous significant voxels) to produce activation cluster maps. The combination of probability and cluster thresholding was used to maximize the power of the statistical test while holding the likelihood of false positives to a minimum. 3dClustSim was used to determine the cluster threshold. The program takes group-averaged EPI mask parameters, the spatial correlation of voxels (must be contiguous on three sides), and the voxelwise threshold as input and runs a series of Monte Carlo simulations (10,000 iterations for our study) to determine the frequency of clusters of varying sizes produced by chance. From this frequency distribution, we then selected the cluster size (162 $\mu$ l, given our parameters) that occurs $<1 \%$ of the time by chance, to give a threshold of $p=.01$ (corrected). Corrected activation maps for correct money trials were determined using the same parameters.

For the purposes of functionally defined ROI analyses, activation cluster maps from whole-brain analyses for correct DR-IP and neutral no-go events were computed to create an overall correct no-go map that included voxels of activation indicated as significant from either of the constituent maps. Mean activations from clusters within the correct no-go map were then calculated for both DR-IP and neutral no-go events. We were also interested in activity occurring during correct money trial responses in the same group of clusters. Thus, mean activations from clusters within the correct no-go map were also calculated for DR-IP and neutral money events. Derived mean activations were analyzed using separate repeated-measures 2 (trial type) $\times 2$ (task condition) ANOVA for each cortical node, corrected via a modified Bonferroni's procedure for multiple comparisons (Keppel, 1991).

\section{FFA Localization}

To localize face-sensitive activity in the fusiform gyrus, a separate analysis of task data that employed go trial events was run as the sole regressor-of-interest. This procedure enabled face-related activity to be derived from within-task events that were unrelated to primary task regressors-ofinterest. Preprocessing steps were run in the same way as our primary analysis. To avoid baseline contamination, 
no-go and money trial errors and motion were modeled as additional regressors. Because feedback screen events did not include presentations of faces, mean activation for these events were utilized as the baseline estimate. An activation cluster map for go events was derived using the same thresholding and clustering parameters as our primary analysis. Mean activations from this cluster were then calculated for DR-IP and neutral no-go and money events.

\section{RESULTS}

\section{Behavioral Results}

Performance indices from the DR-IP go/no-go task are presented in Figure 2. Repeated-measures $t$ tests demonstrated significant improvements in performance under DR-IP task conditions for both inhibitory control accuracy (79\% vs. $69 \%, t(16)=2.6, p=.01)$ and number of consecutive successful inhibitions (9.3 vs. 6.9, $t(16)=2.2$, $p=.04)$. The absence of concurrent slowing in DR-IP response speeds for both go trials (DR-IP $=378 \mathrm{msec}$ vs. neutral $=372 \mathrm{msec}, t(16)=0.6, p=.53)$ and money trials $(\mathrm{DR}-\mathrm{IP}=396 \mathrm{msec}$ vs. neutral $=400 \mathrm{msec}, t(16)=$ $0.6, p=.65$ ) indicates that enhanced DR-IP inhibition performance was not achieved via a speed-accuracy trade-off and rather implies more efficient inhibitory control performance with DR-IP contingencies. In addition, the lack of correlation between inhibitory control accuracy and both go trial response time (RT; $r=.01, p=.95$ ) and money trial RT $(r=.2, p=.43)$ for DR-IP is suggestive of an absence of a speed-accuracy trade-off at the individual level. We also tested for the possibility that improvements in DR-IP inhibitory control were associated with slowed go RTs as participants accumulated consecutive inhibitions and DRs grew. RTs for go trials before the first successfully inhibited no-go trial of a consecutive run were compared with RTs for go trials before the last successfully inhibited no-go trial of a consecutive run for each condition (DR-IP and neutral). Using a 2 (go RT before the first and last successful inhibitions of a consecutive run) $\times 2$ (DR-IP and neutral) ANOVA, we found no significant main effects of go RT before the first and last successful inhibitions of a consecutive run (first go RT $=385 \mathrm{msec}$ vs. last go $\mathrm{RT}=375 \mathrm{msec}, F(1,16)=0.94, p=.76)$ or condition $(\mathrm{DR}-\mathrm{IP}=380 \mathrm{msec}$ vs. neutral $=381 \mathrm{msec},(F(1,16)=$ $1.45, p=.24)$. Moreover, there was no evidence of an interaction between go RT before the first and last successful inhibitions of a consecutive run and condition $(F(1,16)=$ $0.88, p=.36)$. Differences in go trial and money trial response speeds for each task condition were examined using a 2 (trial type) $\times 2$ (task condition) repeatedmeasures ANOVA. A significant main effect of trial type was found $(F(1,16)=27.8, p=.0001)$, with participants responding faster for go trials when compared with money trials. Sensitivity to Punishment and Sensitivity to Reward Questionnaire self-report scores for SR and SP subscales were $11(S D=5.89)$ and $9.12(S D=4.40)$, respectively. Consistent with previous findings, SP and SR scores were not significantly correlated $(r=.07, p=.77)$.

\section{Imaging Results}

\section{Whole-brain Event-related Activity for Successful No-go Trials}

The event-related functional analysis of BOLD activity associated with successful inhibitory control revealed

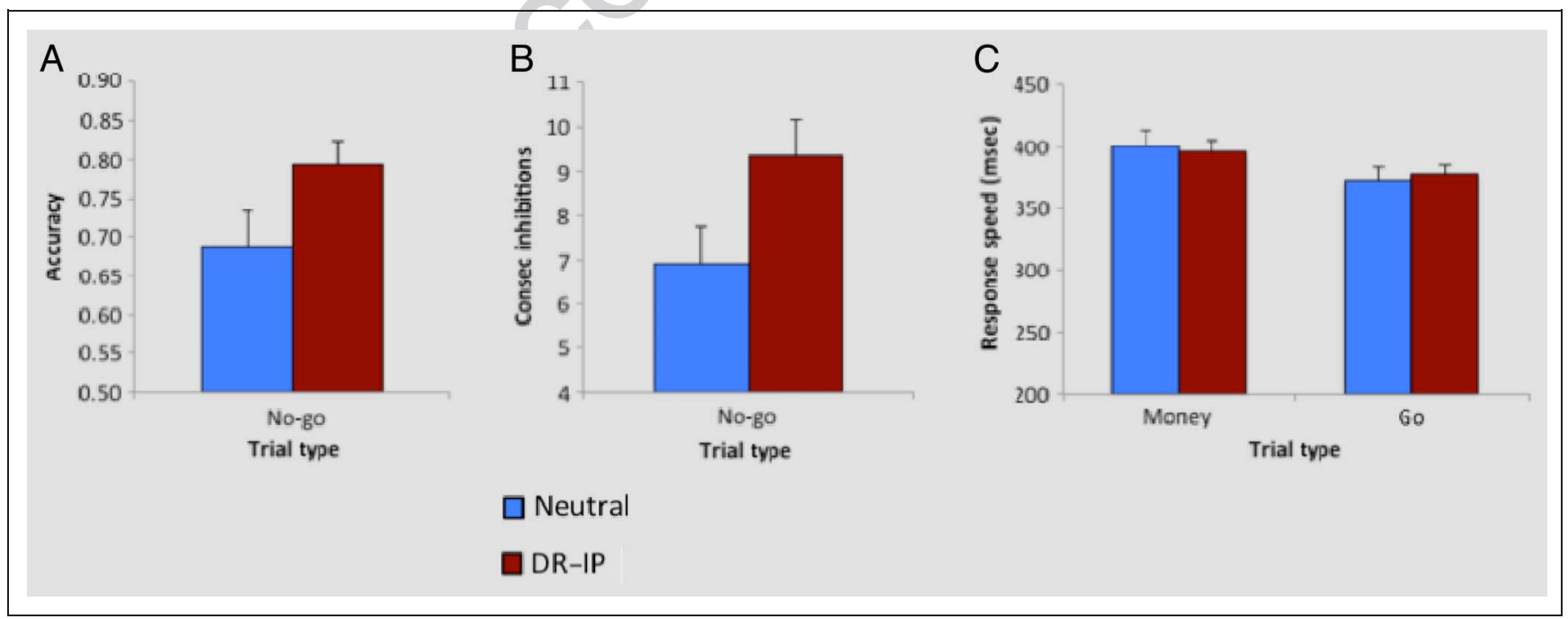

Figure 2. Behavioral performance on the DR-IP go/no-go task. (A) Inhibitory control accuracy proportions as a function of task condition: DR-IP and neutral. A significant improvement for DR-IP inhibitory control was present (79\% vs. 69\%). (B) Average number of consecutive successful inhibitions per run as a function of task condition: DR-IP and neutral. DR-IP consecutive inhibitions were significantly higher (9.3 vs. 6.9). (C) Response times for each trial type (money trials and go trials) as a function of task condition: DR-IP and neutral. No speed difference between task conditions was present for either money trials (DR-IP $=396 \mathrm{msec} v s$. neutral $=400 \mathrm{msec})$ or go trials (DR-IP $=378 \mathrm{msec}$ vs. neutral $=372 \mathrm{msec}$ ). Participants responded significantly faster for go trials compared with money trials. Error bars indicate standard error (SE). 
13 clusters of activity (Table 1 ). These included four frontal regions that have previously been associated with inhibitory control. Extensive activity was observed in a ventral location of the rIFG extending into the anterior insula (Figure 3C). A smaller cluster was revealed in the rIFJ, a dorsal IFG region extending into the right precentral gyrus (Figure 3A). These are consistent with previous findings regarding key nodes of the right IFC engaged in inhibitory control performance (Barber, Caffo, Pekar, \& Mostofsky, 2013; Boehler et al., 2011; Cai \& Leung, 2011; Chikazoe et al., 2009; Hampshire, Thompson, Duncan, \& Owen, 2009; Hodgson et al., 2007; Leung \& Cai, 2007; Aron, 2006; Li, Huang, Constable, \& Sinha, 2006; Kelly et al., 2004; Aron et al., 2003; Menon, Adleman, White, Glover, \& Reiss, 2001; Rubia et al., 2001; Garavan et al., 1999; Konishi et al., 1999; Kawashima et al., 1996), as is the right medial pre-SMA, which also yielded a large cluster of activity (Figure 3B; Chen, Scangos, \& Stuphorn, 2010; Sharp et al., 2010; Duann, Ide, Luo, \& Li, 2009; Nachev, Kennard, \& Husain, 2008; Picton et al., 2007; Sumner et al., 2007; Floden \& Stuss, 2006; Li et al., 2006). Consistent with a number of studies (Criaud \& Boulinguez, 2013; Hirose et al., 2012; Zhang \& Li, 2012; Swick, Ashley, \& Turken, 2008; Rubia et al., 2001), successful inhibitory control was associated with left-lateralized activity in a ventral region of the IFG extending into the anterior insula (Figure 3D). Activation was also found in an area of the right STG, which has previously shown sensitivity to task-relevant stop trials (Boehler et al., 2011).

Table 1. Regions of Event-related Activation during Successfully Inhibited No-go Trials

\begin{tabular}{lrrrr}
\hline & & \multicolumn{3}{c}{ MNI Coordinates } \\
\cline { 3 - 5 } Brain Region & Volume $(\mu l)$ & $x$ & $y$ & $z$ \\
\hline R inferior frontal/insula & 4471 & 35 & 19 & 7 \\
L postcentral & 4164 & -41 & -28 & 51 \\
R pre-SMA & 4154 & 9 & 2 & 56 \\
L inferior frontal/insula & 4119 & -31 & 18 & 9 \\
L precentral & 520 & -30 & -11 & 53 \\
R inferior frontal/precentral & 509 & 43 & -2 & 37 \\
R superior temporal & 507 & 49 & -47 & 15 \\
L superior frontal & 392 & -17 & -7 & 69 \\
L angular & 259 & -47 & -68 & 28 \\
L middle frontal & 223 & -29 & 39 & 29 \\
L inferior frontal & 219 & -40 & 36 & -7 \\
R cuneus & 196 & 14 & -71 & 30 \\
L middle cingulate & 174 & -6 & 18 & 32 \\
\hline
\end{tabular}

$\mathrm{R}=$ right; $\mathrm{L}=$ left.

\section{ROI Task-related Activity}

Our primary aim was to ascertain the role played by key cortical nodes implicated in inhibitory control during DR-IP action withholding and how the same regions responded during alluring money trials. The relevance of the latter aim is based on proposed alternative roles of regions implicated in inhibitory control such as attentional detection and context monitoring. To examine the association between changes in BOLD activity in four key frontal regions (bilateral IFG/insula, right pre-SMA, and rIFJ/ precentral gyrus) and the observed improvement in DRIP action withholding, we subjected them to a 2 (trial type) $\times 2$ (task condition) ANOVA. Given its engagement in relevant literature, we also included the right STG in our analyses. Of the four frontal clusters analyzed (Figure 3), all except the rIFJ/precentral gyrus displayed qualitatively similar patterns of activity. That is, three areas (rIFG and left IFG/insula, right pre-SMA) produced significantly more activity during correctly withheld no-go trials than during motor responses to money trials (rIFG/insula, $p<.001$; left IFG/insula, $p<.001$; right pre-SMA, $p<.001$ ). We also observed significant interaction effects bilaterally in IFG/ insula clusters (rIFG/insula, $p=.013$; left IFG/insula, $p=$ .006). Post hoc comparisons of differences between trials in each task condition showed that the interaction effect in both IFG/insula clusters was driven by a nonsignificant reduction in activity on money trials in the DR-IP condition compared with the neutral task condition (rIFG/insula, $p=$ .15 ; left IFG/insula, $p=.14$ ), which was not observed between DR-IP and neutral no-go trials (rIFG/insula, $p=$ .61 ; left IFG/insula, $p=.74$ ). To verify that patterns of activity in the IFG did not differ bilaterally, we submitted both regions to an ANOVA (2, laterality $\times 2$, trial type $\times 2$, task condition). As expected, the previously reported main effect $(p<.001)$ and interaction $(p=.007)$ were found, but no interaction effect related to laterality was present $(p=.19)$.

A significant interaction between trial type and task condition was also found in the rIFJ/precentral gyrus ( $p=$ .012; Figure 3A). The pattern of results in this cluster was qualitatively different from that seen in the IFG/insula clusters. Activity in the rIFJ/precentral gyrus was found to be higher for money trials compared with no-go trials in the neutral task condition $(p=.005)$ but not in the DRIP task condition $(p=.86)$. Moreover, activity was significantly higher for no-go trials in the DR-IP task condition when compared with the neutral task condition $(p=$ .028). Of the additional nonfrontal cluster submitted to statistical analysis (right STG), we observed an interaction effect that showed higher activity for no-go trials during the DR-IP task condition; however, this effect did not survive Bonferroni's adjustment $(p=.032)$.

\section{Successful Money Trial Activity}

Clusters of activity during correct responses to rewarding money trials can be found in Table 2. Using the same 


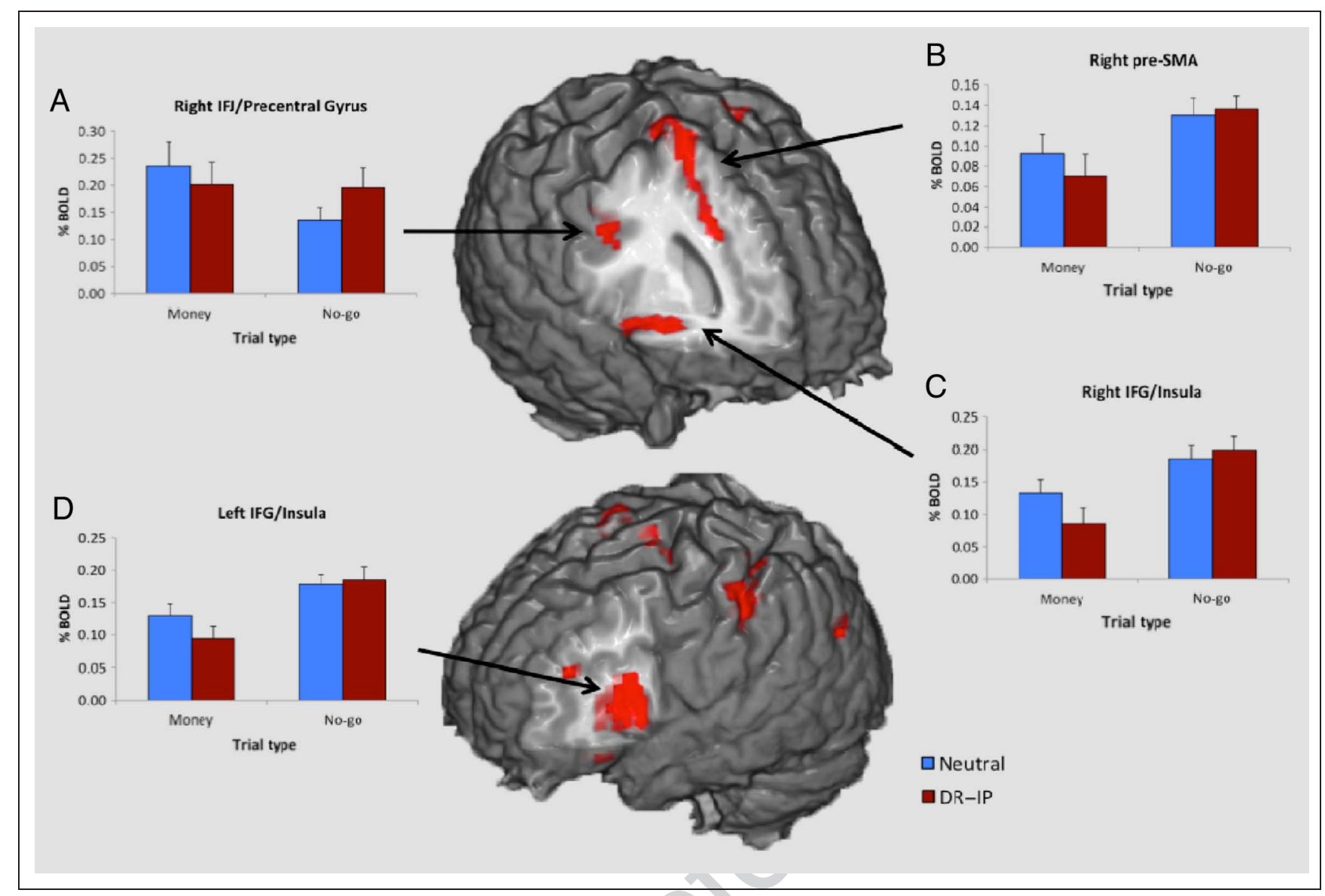

Figure 3. Frontal brain BOLD activity clusters functionally derived from successfully inhibited no-go trials. Graphs display activity during both money trials and no-go trials as a function of task condition: DR-IP and neutral. Clusters encompassing rIFG (C; Montreal Neurological Institute [MNI] coordinates: $x=35, y=19, z=7$ ) and left IFG (D; MNI coordinates: $x=-31, y=18, z=9)$ or anterior insula demonstrated comparable significant interaction effects between trial type (money, go) and DR-IP task condition (DR-IP, neutral). The same clusters and a cluster in the right pre-SMA (B; MNI coordinates: $x=9, y=2, z=56$ ) demonstrated a main effect in activity for no-go trials over money trials. A cluster encompassing the rIFJ and precentral gyrus (A; MNI coordinates: $x=43, y=-2, z=37$ ) also produced a significant interaction effect between trial type (money, go) and DR-IP task condition (DR-IP, neutral). Error bars indicate $S E$.

thresholding and clustering parameters that were applied to inhibitory control activity, we found extensive clusters of activation covering both left and right fusiform gyri and middle occipital gyrus. In addition to bilateral activity in the inferior frontal and cingulate gyri, we observed subcortical activation in the caudate and right thalamus. Striatal caudate activity has previously been associated with the reinforcement of action potentially leading to reward (Tricomi, Delgado, \& Fiez, 2004) and also proactive motor suppression (Majid, Cai, Corey-Bloom, \& Aron, 2013).

Given these associations and the general pattern of greater activity for no-go trials in frontal clusters, we also examined whether this striatal cluster showed a different profile of sensitivity to task condition and trial type (Figure 4). A $2 \times 2$ ANOVA revealed trends for main effects for both trial type and task condition. For trial type, subcortical activity was suggestive of a reversal of the overall pattern observed in frontal clusters such that activity was higher for money trials compared with no-go trials ( $p=$ .056). In addition, there was also a trend for greater activity in the neutral task condition compared with the DR-IP task condition $(p=.069)$.

\section{Correlations with Self-reported Punishment and Reward Sensitivity}

We examined whether activity in regions implicated in inhibitory control correlated with self-reported measures of SR and punishment. Correlational analyses between both SR and SP scores and key clusters of activity (rIFG and left IFG/insula, right pre-SMA, and rIFJ/precentral gyrus) during both no-go trials and money trials revealed one significant correlation. Individual differences in selfreported SP were found to be positively correlated with responsivity in the rIFJ/precentral gyrus during DR-IP action withholding $(r=.55, p=.02$; Figure 5); however, this effect did not survive the Bonferroni's adjustment for multiple correlations.

We also examined whether indices of behavioral performance correlated with SP and SR scores. Correlational analyses between both scores and indexes of behavioral 
Table 2. Regions of Event-related Activation during Correct Responses for Money Trials

\begin{tabular}{|c|c|c|c|c|}
\hline \multirow[b]{2}{*}{ Brain Region } & \multirow[b]{2}{*}{ Volume $(\mu l)$} & \multicolumn{3}{|c|}{ MNI Coordinates } \\
\hline & & $x$ & $y$ & $z$ \\
\hline $\mathrm{R}$ fusiform & 18039 & 34 & -66 & -14 \\
\hline L fusiform & 16274 & -36 & -70 & -12 \\
\hline $\mathrm{R}$ inferior frontal & 7362 & 40 & 14 & 28 \\
\hline $\mathrm{R}$ middle occipital & 4560 & 30 & -63 & 37 \\
\hline $\mathrm{L}$ inferior parietal & 4386 & -30 & -58 & 39 \\
\hline L precentral & 3043 & -43 & 2 & 34 \\
\hline $\mathrm{L}$ insula & 1532 & -29 & 17 & 14 \\
\hline L middle frontal & 1218 & -44 & 24 & 33 \\
\hline R SMA & 1002 & 5 & 6 & 54 \\
\hline $\mathrm{R}$ thalamus & 721 & 16 & -29 & -3 \\
\hline $\mathrm{L}$ middle frontal & 650 & -28 & 22 & 50 \\
\hline $\mathrm{L}$ inferior/middle frontal & 622 & -39 & 43 & 16 \\
\hline L precentral & 476 & -33 & -11 & 49 \\
\hline L posterior cingulate & 363 & -2 & -34 & 30 \\
\hline L middle cingulate & 339 & -5 & -4 & 32 \\
\hline R caudate & 311 & 11 & 9 & 6 \\
\hline $\mathrm{R}$ thalamus & 303 & 9 & -14 & 9 \\
\hline $\mathrm{R}$ middle cingulate & 168 & 6 & -4 & 31 \\
\hline
\end{tabular}

performance (no-go accuracy, go trial RT, and money trial RT) revealed a strong association between SP sensitivity and go trial performance in the DR-IP condition $(r=$ $.68, p=.003)$ and a weaker association between SP sensitivity and money trial performance in the same condition $(r=.49, p=.045)$. There were no correlations between SR sensitivity and indexes of behavioral performance.

\section{Task-related Activity in a Localized FFA Cluster}

We used a functionally derived face-sensitive cluster in the right fusiform gyrus to examine the potential for changes in activity related to attentional modulation at the visual processing level. FFA localization produced a cluster $($ size $=466 \mu \mathrm{l})$ with Talairach coordinates $(x=37, y=$ $-46, z=-14$ ) in an approximately comparable vicinity with those previously reported (e.g., $x=40, y=-55$, $z=-10$; Kanwisher, McDermott, \& Chun, 1997). When activity captured in this region was subjected to a 2 (trial type) $\times 2$ (task condition) ANOVA, we observed a main effect for trial type whereby activity was significantly higher for money trials when compared with no-go trials $(p<.001)$.

\section{DISCUSSION}

The results of this study demonstrate the relative contributions of key neural nodes implicated in self-control during a motivationally significant and meaningful act of action withholding. We incorporated contingencies in a go/no-go task so that, if successful, inhibition could contribute to a larger monetary reward with delayed feedback (DR) but, if unsuccessful, would result in immediate monetary punishment (IP). Compared with a neutral condition without such implications, we observed a considerable (10\%) improvement in inhibitory control without any corresponding slowing of responses for either go or money trials. Crucially, although regions previously implicated in response inhibition such as rIFG/insula and pre-SMA supported overall action withholding, this behavioral enhancement was reflected by a physiological change in
Figure 4. Striatal BOLD activity clusters functionally derived from successful responses for money trials. Graph displays activity during both money trials and no-go trials as a function of task condition: DR-IP and neutral. A cluster encompassing the right caudate (MNI coordinates: $x=11$, $y=9, z=6$ ) demonstrated main effect trends for both trial type (money trials $>$ no-go trials) and task condition (neutral $>$ DR-IP). Error bars indicate $S E$.

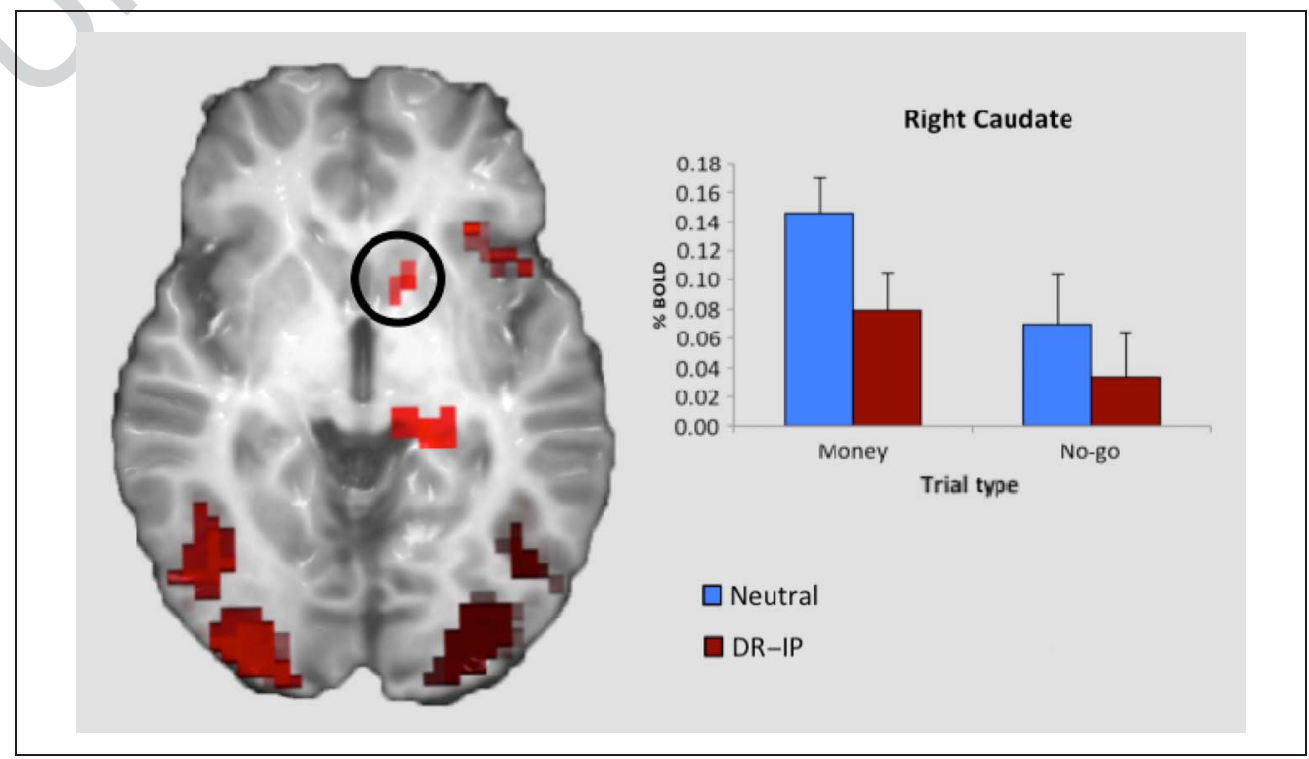




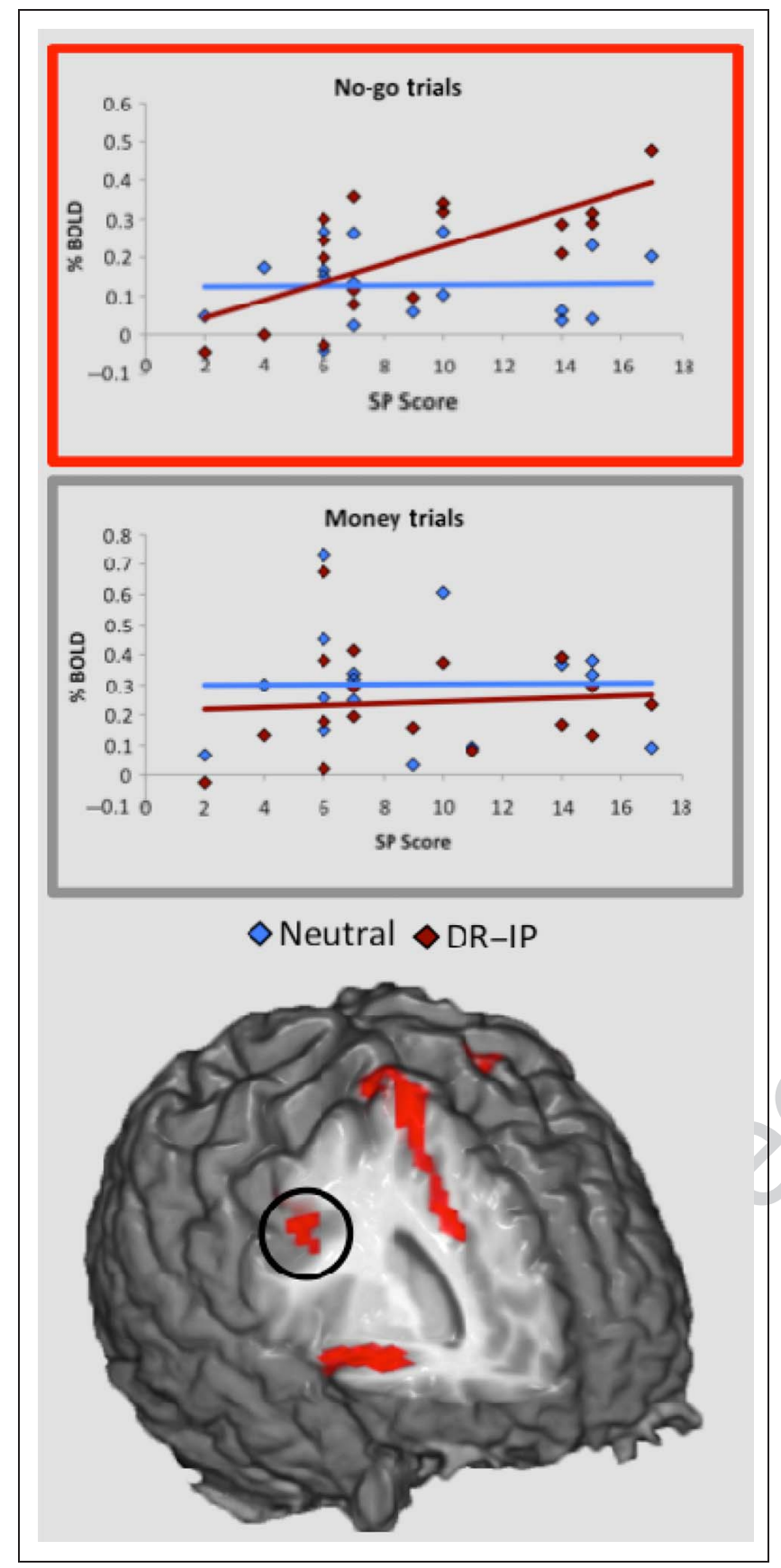

Figure 5. Pearson correlation coefficient scatterplots displaying relationships between self-reported SP and BOLD activity for both no-go and money trials under DR-IP and neutral task conditions. A positive correlation (uncorrected) was found between SP score and activity for DR-IP no-go trials only $(r=.55)$.

a cluster encompassing the rIFJ and precentral gyrus, with greater activity for no-go trial in the DR-IP task condition compared with the neutral task condition.

Recent computational work has shown that the suppression of an impending motor plan may not be wholly dependent on an inhibition trigger but rather by the interplay between perceptual and motor planning processes, such that the success of response inhibition is modified by the outcome of rapid sensory detection (Salinas \& Stanford, 2013). This substantiates the proposal that control-modulating factors such as fluctuations in the attentional/perceptual processing of task-relevant stimuli, rather than stopping processes per se, may be critical to determining improved response inhibition outcomes (Boehler et al., 2011). The rIFJ has been associated with the visual selection of behaviorally relevant stimulus (Verbruggen, Aron, Stevens, \& Chambers, 2010) and is thought to be pivotal to the coordination of stimulusdriven and goal-directed attention (Corbetta \& Shulman, 2011; Asplund et al., 2010; Brass et al., 2005). The region has also been prominent in previous studies involving response inhibition (Barber et al., 2013; Boehler et al., 2011; Cai \& Leung, 2011; Chikazoe et al., 2009; Hampshire et al., 2009) and has also been found to be more active during successful compared with failed stopping (Boehler et al., 2011). The present results implicate this region as critical to the improvement of response inhibition under DR-IP contingencies. Our finding of modulated rIFJ activity, not just for no-go trials but also for immediately rewarding money trials that required responses, favors an interpretation that this region plays a general role in the representation and enforcement of task rules (Boehler, Hopf, Stoppel, \& Krebs, 2012). Results are also convergent with evidence suggesting that the rIFJ may guide response inhibition via stimulus-driven reorientation of attention (Cai \& Leung, 2011; Verbruggen et al., 2010).

Furthermore, the present pattern of results suggests that the rIFJ can be driven by sensitivity to motivational salience not just for current trials, as the association between increased activity and improved DR-IP response inhibition suggests, but also in relation to other types of trials. This kind of context sensitivity in the rIFJ (see also Chatham et al., 2012) is implied by the finding that, although reward implications for money trial responses were consistent between task conditions, we saw modulation in this cluster as a consequence of the monetary implications associated with no-go trials. Thus, within a context in which there were no important implications for failed response inhibition (i.e., neutral, no DR-IP contingencies), the rIFJ/precentral gyrus was at its most active for money trials. Whereas, when presented within a context of potentially punishing DR-IP no-go trials, we observed a reduction in money trial activity. When considered alongside no-go trial activity in this cluster, the overall effect suggests a capacity for dynamic readjustment of task priorities. With the introduction of DR-IP contingencies, a shift in activity for money and no-go trials is elicited so that both are attended to (Cai \& Leung, 2011; Verbruggen et al., 2010) or represented and enforced (Boehler et al., 2011) with equivalent weighting. The finding that increases in rIFJ/precentral gyrus activity for DR-IP no-go trials were positively correlated, albeit uncorrected for multiple comparisons, with levels of self-reported SP appears to favor an interpretation based around attentional sensitivity to motivational salience. 
As discussed, behavioral improvement in the current study appears to be driven by a region at the junction between attention and action implementation. An absence of any influence of DR-IP task conditions on the FFA suggests that regions associated solely with attention are not critical to this enhancement. At the other end of the spectrum, we found no differences in regions more closely associated with action implementation, such as the rIFG and pre-SMA, to suggest an association with improvements in DR-IP action withholding performance. Regarding rIFG functioning, results were supportive of the idea that, in addition to outright stopping, this region plays a role in pausing and "braking" responses (Aron et al., 2014). Specifically, although rIFG activity was at its most elevated during successfully inhibited no-go trials, it also showed sensitivity to money trials requiring motor responses. Previous studies that have employed similar additional go-type trials have demonstrated RTs to be slower than go trials (Chatham et al., 2012; Dodds, Morein-Zamir, \& Robbins, 2011). Importantly, these trials also produced rIFG activity despite the fact that they did not require a stop from participants. This has recently been interpreted to demonstrate a "braking" effect in which action might be paused before being restarted (Aron et al., 2014). Although we anticipated that the incentivized nature of money trials would reduce RTs compared with go trials, response speeds also showed this same pattern of slowing. Moreover, similar to previous studies, this was accompanied by rIFG activity. Although the implementation of money trials represents a limitation of this study, the finding of rIFG activity during trials requiring a response provides support for the claim that any stimulus that is salient, frequent, or unexpected will likely recruit inhibition processes (Aron et al., 2014).

\section{Implications}

In this study, we aimed to examine how people exert inhibitory control over stimuli with response-reward associations when the outcome has implications closer to those in the real world. We found an association between behavioral improvement under these conditions and activity in a region implicated in the coordination of stimulusdriven and goal-directed attention. In light of this finding, we might speculate that inhibitory control dysfunction in clinical conditions such as substance use disorder may be compounded by factors other than dysfunction in behavioral stopping per se. The absence of rIFG involvement in the current study should not discount the important role that rIFG disruption likely plays in impulse control disorders (Whelan et al., 2012; Tabibnia et al., 2011). However, our proposal is consistent with a recent suggestion that individuals with impulse control disorders may have disruptions across multiple neural nodes (Aron et al., 2014). Moreover, a recent computational model of inhibitory control has showed that deficits characterized by response inhibition could be explained by factors such as salience, conflict detection, and motivation and their corresponding neural mechanisms (Wiecki \& Frank, 2013). Such a view is compatible with the incentive sensitization theory (Robinson \& Berridge, 1993, 2008), which states that a major influence on the culmination of the core symptoms of addiction is the biasing of attentional processing towards drug-associated stimuli caused by incentive sensitization. The incentive sensitization theory posits that stimulus incentive salience can vary depending on factors such as the internal state of the perceiver and the degree of activation of the dopamine systems that mediate incentive salience. Within such a framework, our findings of context-sensitive rIFJ activity, and an associated improvement in performance, may be characteristic of a healthy ability to flexibly align stimulusresponse priorities with changing environmental and motivationally significant circumstances. Future studies, in addition to replicating present findings, might investigate whether pathological and compulsive patterns of drugseeking behaviors may be linked to dysfunction in this mechanism.

\section{Acknowledgments}

This research was supported by Australian Research Council Grant DP1092852 and Fellowship (FT1101000088, R. H.) and National Health and Medical Research Grant (628495). The assistance of Michael Kean, Murdoch Children's Research Institute, Flemington, is gratefully acknowledged.

Reprint requests should be sent to Robert Hester, Melbourne School of Psychological Sciences, Redmond Barry Building, University of Melbourne, Melbourne, Victoria 3010, Australia, or via e-mail: hesterr@unimelb.edu.au.

\section{REFERENCES}

Aron, A. R. (2006). Cortical and subcortical contributions to stop signal response inhibition: Role of the subthalamic nucleus. Journal of Neuroscience, 26, 2424-2433.

Aron, A. R., Fletcher, P. C., Bullmore, T., Sahakian, B. J., \& Robbins, T. W. (2003). Stop-signal inhibition disrupted by damage to right inferior frontal gyrus in humans. Nature Neuroscience, 6, 115-116.

Aron, A. R., Robbins, T. W., \& Poldrack, R. A. (2014). Inhibition and the right inferior frontal cortex: One decade on. Trends in Cognitive Sciences, 18, 1-9.

Asplund, C. L., Todd, J. J., Snyder, A. P., \& Marois, R. (2010). A central role for the lateral prefrontal cortex in goal-directed and stimulus-driven attention. Nature Publishing Group, 13, 507-512.

Barber, A. D., Caffo, B. S., Pekar, J. J., \& Mostofsky, S. H. (2013). Effects of working memory demand on neural mechanisms of motor response selection and control. Journal of Cognitive Neuroscience, 25, 1-14.

Boehler, C. N., Appelbaum, L. G., Krebs, R. M., Chen, L.-C., \& Woldorff, M. G. (2011). The role of stimulus salience and attentional capture across the neural hierarchy in a stop-signal task. PLoS ONE, 6, e26386.

Boehler, C. N., Hopf, J.-M., Stoppel, C. M., \& Krebs, R. M. (2012). Motivating inhibition-Reward prospect speeds up response cancellation. Cognition, 125, 498-503. 
Brass, M., Derrfuss, J., Forstmann, B., \& Cramon, D. (2005). The role of the inferior frontal junction area in cognitive control. Trends in Cognitive Sciences, 9, 314-316.

Cai, W., \& Leung, H.-C. (2011). Rule-guided executive control of response inhibition: Functional topography of the inferior frontal cortex. PLOS ONE, 6, e20840.

Chatham, C. H., Claus, E. D., Kim, A., Curran, T., Banich, M. T., \& Munakata, Y. (2012). Cognitive control reflects context monitoring, not motoric stopping, in response inhibition. PLOS ONE, 7, e31546.

Chen, X., Scangos, K. W., \& Stuphorn, V. (2010). Supplementary motor area exerts proactive and reactive control of arm movements. The Journal of Neuroscience, 30, 14657-14675.

Chikazoe, J., Jimura, K., Asari, T., Yamashita, K.-I., Morimoto, H., Hirose, S., et al. (2009). Functional dissociation in right inferior frontal cortex during performance of go/no-go task. Cerebral Cortex, 19, 146-152.

Corbetta, M., \& Shulman, G. L. (2011). Spatial neglect and attention networks. Annual Review of Neuroscience, 34 , 569-599.

Cox, R. W. (1996). AFNI: Software for analysis and visualization of functional magnetic resonance neuroimages. Computers and Biomedical Research, 29, 162-173.

Criaud, M., \& Boulinguez, P. (2013). Have we been asking the right questions when assessing response inhibition in go/no-go tasks with fMRI? A meta-analysis and critical review. Neuroscience \& Biobehavioral Reviews, 37, $11-23$.

Dodds, C. M., Morein-Zamir, S., \& Robbins, T. W. (2011). Dissociating inhibition, attention, and response control in the frontoparietal network using functional magnetic resonance imaging. Cerebral Cortex, 21, 1155-1165.

Duann, J.-R., Ide, J. S., Luo, X., \& Li, C.-S. R. (2009). Functional connectivity delineates distinct roles of the inferior frontal cortex and presupplementary motor area in stop signal inhibition. The Journal of Neuroscience, 29, 10171-10179.

Egner, T., \& Hirsch, J. (2005). Cognitive control mechanisms resolve conflict through cortical amplification of task-relevant information. Nature Neuroscience, 8, 1784-1790.

Fillmore, M. T., \& Rush, C. R. (2002). Impaired inhibitory control of behavior in chronic cocaine users. Drug and Alcohol Dependence, 66, 265-273.

Floden, D., \& Stuss, D. T. (2006). Inhibitory control is slowed in patients with right superior medial frontal damage. Journal of Cognitive Neuroscience, 18, 1843-1849.

Forstmann, B. U., Jahfari, S., Scholte, H. S., Wolfensteller, U., van den Wildenberg, W. P., \& Ridderinkhof, K. R. (2008). Function and structure of the right inferior frontal cortex predict individual differences in response inhibition: A model-based approach. The Journal of Neuroscience, 28, 9790-9796.

Garavan, H., Ross, T. J., \& Stein, E. A. (1999). Right hemispheric dominance of inhibitory control: An event-related functional MRI study. Proceedings of the National Academy of Sciences, U.S.A., 96, 8301-8306.

Goldstein, R. Z., Craig, A. D. B., Bechara, A., Garavan, H., Childress, A. R., Paulus, M. P., et al. (2009). The neurocircuitry of impaired insight in drug addiction. Trends in Cognitive Sciences, 13, 372-380.

Hampshire, A., Thompson, R., Duncan, J., \& Owen, A. (2009). Selective tuning of the right inferior frontal gyrus during target detection. Cognitive, Affective, \& Behavioral Neuroscience, 9, 103-112.

Hirose, S., Chikazoe, J., Watanabe, T., Jimura, K., Kunimatsu, A. Abe, O., et al. (2012). Efficiency of go/no-go task performance implemented in the left hemisphere. Journal of Neuroscience, 32, 9059-9065.
Hodgson, T., Chamberlain, M., Parris, B., James, M., Gutowski, N., Husain, M., et al. (2007). The role of the ventrolateral frontal cortex in inhibitory oculomotor control. Brain, 130, 1525-1537.

Kanwisher, N., McDermott, J., \& Chun, M. M. (1997). The fusiform face area: A module in human extrastriate cortex specialized for face perception. The Journal of Neuroscience, 17, 4302-4311.

Kawashima, R., Satoh, K., Itoh, H., Ono, S., Furumoto, S., Gotoh, R., et al. (1996). Functional anatomy of go/no-go discrimination and response selection-A PET study in man. Brain Research, 728, 79-89.

Kelly, A. M., Hester, R., Murphy, K., Javitt, D. C., Foxe, J. J., \& Garavan, H. (2004). Prefrontal-subcortical dissociations underlying inhibitory control revealed by event-related fMRI. European Journal of Neuroscience, 19, 3105-3112.

Keppel, G. (1991). Design and analysis: A researcher's bandbook. Englewood Cliffs, NJ: Prentice Hall.

Konishi, S., Nakajima, K., Uchida, I., Kikyo, H., Kameyama, M., \& Miyashita, Y. (1999). Common inhibitory mechanism in human inferior prefrontal cortex revealed by event-related functional MRI. Brain, 122, 981-991.

Kreek, M. J., Nielsen, D. A., Butelman, E. R., \& LaForge, K. S. (2005). Genetic influences on impulsivity, risk taking, stress responsivity and vulnerability to drug abuse and addiction. Nature Neuroscience, 8, 1450-1457.

Leung, H.-C., \& Cai, W. (2007). Common and differential ventrolateral prefrontal activity during inhibition of hand and eye movements. The Journal of Neuroscience, 27, 9893-9900.

Li, C.-S. R., Huang, C., Constable, R. T., \& Sinha, R. (2006). Imaging response inhibition in a stop-signal task: Neural correlates independent of signal monitoring and post-response processing. The Journal of Neuroscience, 26, 186-192.

Majid, D. S. A., Cai, W., Corey-Bloom, J., \& Aron, A. R. (2013) Proactive selective response suppression is implemented via the basal ganglia. Journal of Neuroscience, 33, 13259-13269.

Menon, V., Adleman, N. E., White, C. D., Glover, G. H., \& Reiss, A. L. (2001). Error-related brain activation during a go/no-go response inhibition task. Human Brain Mapping, 12, 131-143.

Nachev, P., Kennard, C., \& Husain, M. (2008). Functional role of the supplementary and pre-supplementary motor areas. Nature Reviews Neuroscience, 9, 856-869.

Neubert, F.-X., Mars, R. B., Buch, E. R., Olivier, E., \& Rushworth, M. F. (2010). Cortical and subcortical interactions during action reprogramming and their related white matter pathways. Proceedings of the National Academy of Sciences, U.S.A., 107, 13240-13245.

O'Craven, K. M., \& Kanwisher, N. (2000). Mental imagery of faces and places activates corresponding stimulus-specific brain regions. Journal of Cognitive Neuroscience, 12, 1013-1023.

Oldfield, R. C. (1971). The assessment and analysis of handedness: The Edinburgh inventory. Neuropsychologia, 9, 97-113.

Picton, T. W., Stuss, D. T., Alexander, M. P., Shallice, T., Binns, M. A., \& Gillingham, S. (2007). Effects of focal frontal lesions on response inhibition. Cerebral Cortex, 17, 826-838.

Robbins, T. W., \& Everitt, B. J. (1999). Drug addiction: Bad habits add up. Nature, 398, 567-570.

Robinson, T. E., \& Berridge, K. C. (1993). The neural basis of drug craving: An incentive-sensitization theory of addiction. Brain Research Reviews, 18, 247-291.

Robinson, T. E., \& Berridge, K. C. (2008). The incentive sensitization theory of addiction: Some current issues. 
Philosophical Transactions of the Royal Society, Series B, Biological Sciences, 363, 3137-3146.

Rubia, K., Russell, T., Overmeyer, S., Brammer, M. J., Bullmore, E. T., Sharma, T., et al. (2001). Mapping motor inhibition: Conjunctive brain activations across different versions of go/no-go and stop tasks. Neuroimage, 13, 250-261.

Salinas, E., \& Stanford, T. R. (2013). The countermanding task revisited: Fast stimulus detection is a key determinant of psychophysical performance. Journal of Neuroscience, 33, 5668-5685.

Salo, R., Nordahl, T. E., Possin, K., Leamon, M., Gibson, D. R., Galloway, G. P., et al. (2002). Preliminary evidence of reduced cognitive inhibition in methamphetaminedependent individuals. Psychiatry Research, 111, 65-74.

Sava, F. A., \& Sperneac, A.-M. (2006). Sensitivity to reward and sensitivity to punishment rating scales: A validation study on the Romanian population. Personality and Individual Differences, 41, 1445-1456.

Sharp, D. J., Bonnelle, V., De Boissezon, X., Beckmann, C. F., James, S. G., Patel, M. C., et al. (2010). Distinct frontal systems for response inhibition, attentional capture, and error processing. Proceedings of the National Academy of Sciences, U.S.A., 107, 6106-6111.

Sumner, P., Nachev, P., Morris, P., Peters, A. M., Jackson, S. R., Kennard, C., et al. (2007). Human medial frontal cortex mediates unconscious inhibition of voluntary action. Neuron, 54, 697-711.

Swick, D., Ashley, V., \& Turken, A. U. (2008). Left inferior frontal gyrus is critical for response inhibition. BMC Neuroscience, 9, 102 .
Swick, D., Ashley, V., \& Turken, U. (2011). Are the neural correlates of stopping and not going identical? Quantitative meta-analysis of two response inhibition tasks. Neuroimage, 56, 1655-1665.

Tabibnia, G., Monterosso, J. R., Baicy, K., Aron, A. R., Poldrack, R. A., Chakrapani, S., et al. (2011). Different forms of self-control share a neurocognitive substrate. Journal of Neuroscience, 31, 4805-4810.

Torrubia, R., Ávila, C., Moltó, J., \& Caseras, X. (2001). The sensitivity to punishment and sensitivity to reward questionnaire (SPSRQ) as a measure of Gray's anxiety and impulsivity dimensions. Personality and Individual Differences, 31, 837-862.

Tricomi, E. M., Delgado, M. R., \& Fiez, J. A. (2004). Modulation of caudate activity by action contingency. Neuron, 41, 281-292.

Verbruggen, F., Aron, A. R., Stevens, M. A., \& Chambers, C. D. (2010). Theta burst stimulation dissociates attention and action updating in human inferior frontal cortex. Proceedings of the National Academy of Sciences, U.S.A., 107, 13966-13971.

Whelan, R., Conrod, P. J., Poline, J.-B., Lourdusamy, A., Banaschewski, T., Barker, G. J., et al. (2012). Adolescent impulsivity phenotypes characterized by distinct brain networks. Nature Publishing Group, 15, 920-925.

Wiecki, T. V., \& Frank, M. J. (2013). A computational model of inhibitory control in frontal cortex and basal ganglia. Psychological Review, 120, 329-355.

Zhang, S., \& Li, C.-S. R. (2012). Functional networks for cognitive control in a stop signal task: Independent component analysis. Human Brain Mapping, 33, 89-104. 


\section{University Library}

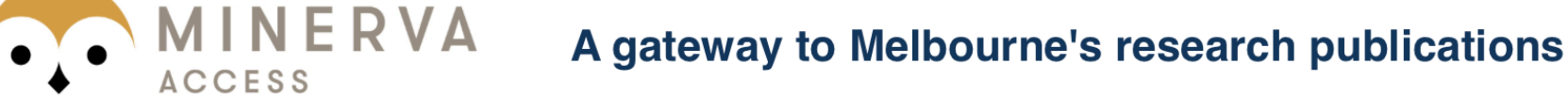

Minerva Access is the Institutional Repository of The University of Melbourne

\section{Author/s:}

O'Connor, DA;Upton, DJ;Moore, J;Hester, R

Title:

Motivationally Significant Self-control: Enhanced Action Withholding Involves the Right Inferior Frontal Junction

\section{Date:}

2015-01-01

\section{Citation:}

O'Connor, D. A., Upton, D. J., Moore, J. \& Hester, R. (2015). Motivationally Significant Self-control: Enhanced Action Withholding Involves the Right Inferior Frontal Junction. JOURNAL OF COGNITIVE NEUROSCIENCE, 27 (1), pp.112-123. https://doi.org/10.1162/ jocn_a_00695.

Persistent Link:

http://hdl.handle.net/11343/116620 